\title{
Análise de Falhas em Materiais Através de Técnicas Avançadas de Microscopia
}

A análise de falhas em materiais depende de técnicas analíticas que possibilitem a determinação da composição química da amostra e correlacioná-la ao modo de fratura observado. A microscopia eletrônica de varredura (MEV) desempenha papel importante no estudo de falhas devido a sua alta resolução, profundidade de foco e utilização de recursos analíticos, que possibilitam a realização in-situ de microanálise ou mapeamento químico da superfície da amostra. Aplicações qualitativas dos recursos da técnica no estudo de fratura de polímeros reforçados com cargas minerais são apresentados. Imagens de SEI - Elétrons secundários - fornecem informações sobre a topografia da amostra (modo de fratura) e BSE - Elétrons retroespalhados - mais sensíveis ao número atômico - sobre a dispersão de cargas na matriz polimérica. A composição química das cargas ou contaminantes responsáveis pela iniciação do processo de fratura é determinada por EDS-energia dispersiva de raios-x.

\section{Introdução}

A incorporação de cargas minerais a polímeros pode ser empregada como enchimento - redução de custos - ou como reforço estrutural. Assim, produtos moldados contendo cargas minerais reforçantes apresentam melhor desempenho, menor encolhimento no molde, além de controle de flamabilidade. Comercialmente existem inúmeras cargas minerais que podem ser utilizadas no reforço de termoplásticos: talco, carbonato de $\mathrm{Ca}$; negro de fumo, sílica, óxido de titânio, microesferas de vidro dentre as mais usadas. Além de atuarem como reforços, algumas cargas minerais particuladas podem agir como nucleantes de matrizes termoplásticas semi-cristalinas (poliamidas, polipropileno), contribuindo para um aumento da rigidez do composto e maximização de sua temperatura de uso. $^{1}$
O desempenho mecânico desses compostos poliméricos está associado à eficiência da dispersão da carga na matriz termoplástica durante o seu processamento. Propriedades como alongamento na ruptura e resistência ao impacto permitem uma avaliação da eficiência do processo de incorporação. O comportamento de fratura pode ser avaliado em produtos acabados ou através de ensaios físicos em corpos de prova. Estudos de fratografia podem ser efetuados utilizando microscopia óptica (MO), no entanto na maioria dos casos as superfícies fraturadas apresentam rugosidade excessiva, limitando assim o uso da MO devido a sua pequena profundidade de foco particularmente em aumentos elevados.

Embora em alguns casos ainda possam ser empregadas réplicas, a utilização de MET restringe-se a casos que requerem resolução de estrutura fina tal como microfibrilação ou cisalhamento em plásticos tenacifi$\operatorname{cados}^{2}$. Na maioria dos casos a resolução do MEV é suficiente para a investigação de falhas envolvendo compostos termoplásticos.

Análises do tipo de fratura podem ser realizadas in-situ, através de dispositivos especiais adaptados ao MEV, em corpos de prova pósensaiados ou para a determinação da causa de falhas de componentes microestruturais em serviço. Nesses casos, imagens formadas por elétrons secundários (SEI) permitem uma observação do tipo de fratura, dúctil ou frágil, e imagens formadas por elétron retroespalhados (BSEI) permitem a obtenção de contraste por peso atômico facilitando a distinção de cargas minerais inorgânicas em matrizes poliméricas. A presença de contaminantes ou má dispersão de cargas pode levar a concentração de tensões e formação de 
"locus" de onde pode-se iniciar uma fratura do tipo frágil.

$\mathrm{Na}$ análise desses defeitos superficiais menores está, muitas vezes, a solução para o problema causador da falha. São micro-trincas superficiais, micro-poros, aglomerados de carga mineral mal disperso, entre outros, que podem vir a servir como pontos de concentração de tensão, e consequentemente de início de falha. No caso de defeitos físicos, nem sempre a imagem por MEV, simplesmente, traz soluções efetivas. A presença de uma partícula grosseira na superfície de fratura pode gerar questionamentos a respeito de sua composição e origem, cujas respostas podem conduzir às reais causas da falha. Tais respostas podem ser alcançadas através de técnicas associadas ao microscópio eletrônico de varredura, como por exemplo a espectrometria por dispersão de energia de raios-x (EDS), um método de análise química elementar de pequenas regiões.

\section{A espectrometria por dispersão de energia de raios-X (EDS)}

Os sinais utilizados na análise química por EDS são gerados a partir da interação de um feixe de elétrons de alta energia $(20 \mathrm{kV}$ de potencial) com os elementos químicos da superfície da amostra. Nesse caso, de tal interação são produzidos fótons de raios-x cujos comprimentos de onda são característicos para cada elemento com o qual houve a interação. Os sinais gerados pela amostra são captados por detectores específicos e apresentados na forma de espectros de energia versus intensidade relativa dos picos. A intensidade relativa dos picos deve levar em conta alguns fatores, uma vez que a geração dos fótons está sujeita a flutuações estatísticas, sendo o valor medido sempre uma média. Uma medida dessa distribuição é dada pelo valor da "largura à meia altura" (LMA) do pico de um determinado elemento, em unidades de energia. Para o sistema EDS esse valor é da ordem de $2,5 \%$ da energia de intensidade máxima do pico. Em muitas situações, essa característica do sistema pode causar problemas de sobreposição de picos, restringindo assim a resolução espectral da técnica.

A grande vantagem da técnica está no fato de que a análise é realizada a partir de um volume muito pequeno de material. Considerando-se a interação do feixe de elétrons com o material, como ilustra a Figura 1, raios-x podem ser gerados a partir de volumes com dimensões lineares em torno de $1 \mu \mathrm{m}$. Isso implica que volumes menores que $10^{-12} \mathrm{~cm}^{3}$ podem ser analisados. Assumindo-se que a densidade típica de um polímero esteja na faixa de $1-2 \mathrm{~g} / \mathrm{cm}^{3}$, a composição de apenas $1-2.10^{-12} \mathrm{~g}$ de material pode ser determinada. A detecção dessa pequena quantidade de massa de amostra deve ser limitada pelo volume de interação do feixe de elétrons. Para elementos com número atômico $Z>10$ o limite de detecção da técnica está entre 10 e 100 ppm³ .

\section{Limite de detecção ${ }^{4}$}

Define-se como sendo Limite de Detecção (LD) a menor fração de um determinado elemento que pode ser detectado considerando-se um determinado intervalo de confiança. O limite de detecção está intimamente relacionado à resolução espacial (volume a ser analisado), de modo que o aumento desse fator implica em uma redução no limite de detecção. Para resoluções espaciais mais elevadas o volume analisado é menor, e consequentemente a intensidade do sinal reduzse. Isso significa que o espectro adquirido conterá uma quantidade maior de ruídos e os picos dos elementos em menores concentrações, serão mais dificilmente detectados. Como exemplo, a Figura 2 mostra as dimensões dos volumes de análise para as técnicas de microanálise em MEV, Microscopia Eletrônica de Transmissão (TEM) e para Microscopia Eletrônica de Varredura e Transmissão utilizando como

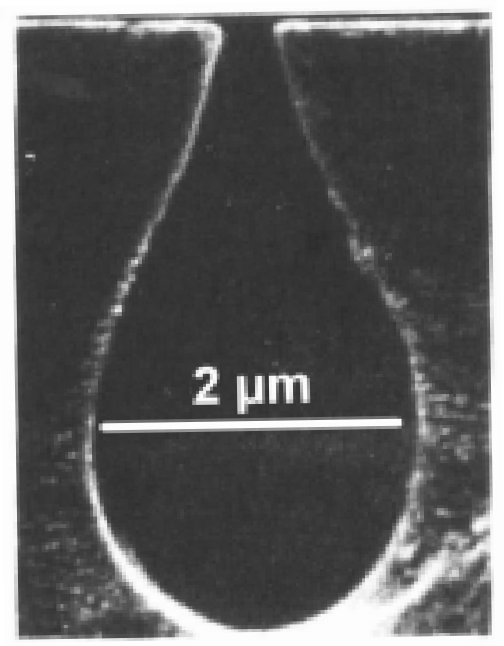

a)

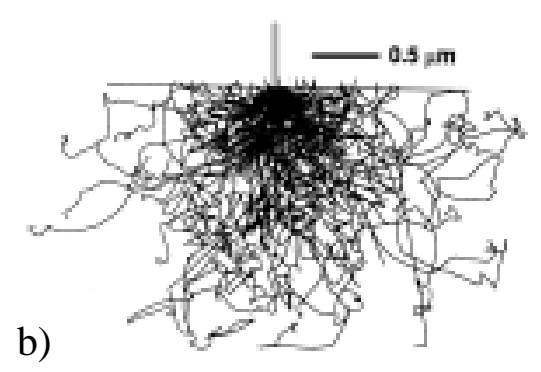

Figura 1. Ilustração da geometria de interação feixe/matriz. (a) Visualização direta em polimetilmetacrilato cujo volume de interação, obtido a partir de um potencial de $20 \mathrm{kV}$, foi revelado através de ataque químico e (b) simulação da trajetória de elétrons pelo método de Monte Carlo em carbono sob um potencial de $20 \mathrm{kV} .^{3}$ 
fonte um "Field Emission Gun" (STEM/FEG).

Dessa forma, pode-se definir o limite de detecção (LD) em termos da mínima fração de massa (em porcentagem em peso ou ppm) que pode ser medida no volume de análise. Esse limite mínimo pode ser relacionado diretamente com os parâmetros de microanálise, de acordo com Ziebold ${ }^{5}$ :

$$
\mathrm{LD} \alpha \frac{1}{\sqrt{\mathrm{P} \frac{\mathrm{P}}{\mathrm{B}} \mathrm{n} \tau}} \quad \text { eq. } 01
$$

onde P é o número de contagens de raios-x para o pico característico do elemento, P/B é a razão entre as contagens do pico e as do ruído de fundo, e $\tau$ é o tempo de análise para cada uma das $n$ análises realizadas.

Para se reduzir LD, ou seja aumentar a capacidade do sistema de microanálise em detectar elementos em pequenas concentrações, pode-se aumentar P através do aumento da corrente do feixe de elétrons. Pode-se também aumentar a relação $\mathrm{P} / \mathrm{B}$ utilizando valores mais elevados de voltagem. Também é recomendado a utilização de um equipamento estável e com vácuo relativamente limpo para eliminar ou reduzir a deterioração e contaminação da amostra. O controle do tempo e do número de análises varia muito de amostra para amostra e também da concentração do elemento considerado. Concentrações muito pequenas podem requerer um tempo grande de análise. Nesses casos, é evidente que o investimento de muito tempo em uma única análise pode ser arriscado, a menos que se tenha escolhido criteriosamente a região de análise, e se tenha certeza que o tempo investido trará bons resultados. Outro fator a conside-

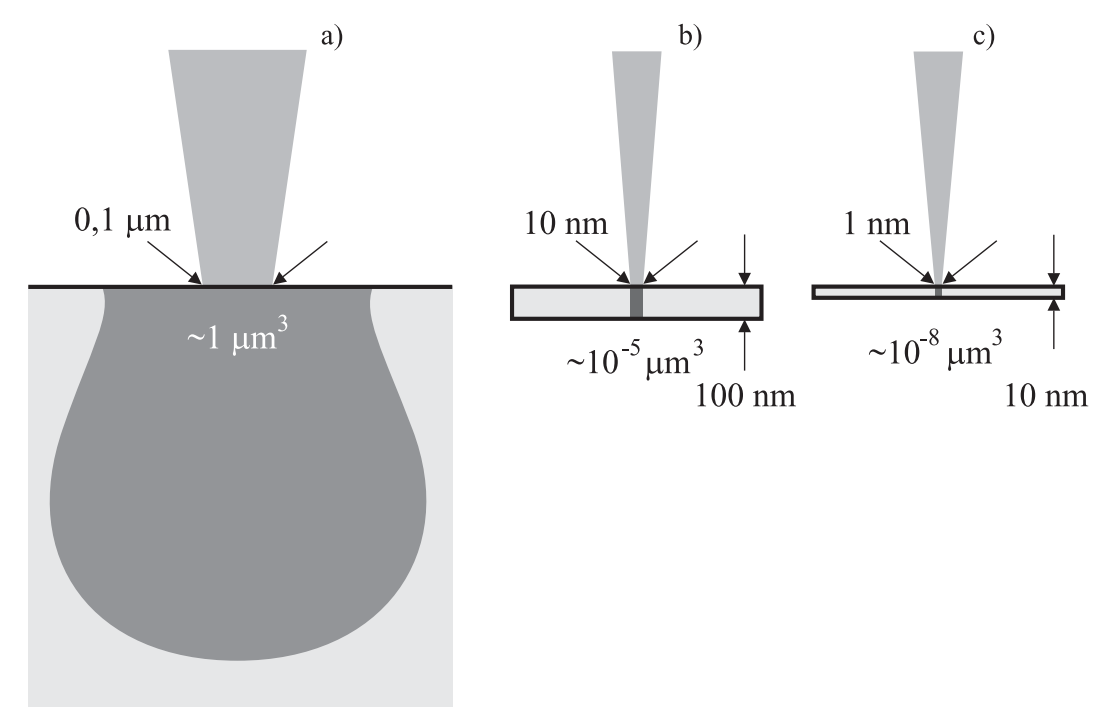

Figura 2. Comparação entre as dimensões relativas do volume de interação feixe-amostra para a) MEV, b) TEM e c) STEM-FEG ${ }^{4}$

rar é a degradação da amostra com o tempo de análise.

Essa capacidade de detecção, considerada até o momento, sugere que tais técnicas de quantificação de elementos sejam técnicas de análise de traços. $\mathrm{O}$ termo traço, como utilizado em química analítica, se refere à capacidade de se detectar uma fração ínfima de um constituinte no volume total de determinada amostra. Geralmente, traço referese a concentrações cujos valores se aproximam do limite de detecção, e, portanto, varia de técnica para técnica. Baseado em observações práticas para a técnica de microanálise por espectrometria por dispersão de energia de raios-x adota-se os seguintes limites ${ }^{3}$ :

Elementos majoritários: > $10 \%$ em peso;

Elementos minoritários: 1-10\% em peso;

Traço: $<1 \%$ em peso

\section{Detetor de elétrons retroespalhados (BSED).}

Além das imagens simplesmente topográficas, ou seja, as obtidas a partir de elétrons de menor energia (elétrons secundários - SE), existem aquelas geradas a partir de elétrons retroespalhados (BSE - back scattered electrons). Esse tipo de imagem traz consigo informações da própria composição química superficial, que quando captadas e interpretadas pelo sistema, fornecem imagens não mais com contraste topográfico e sim com contraste de peso atômico. A imagem por BSE é formada por elétrons que emergem da superfície após consecutivos espalhamentos elásticos essencialmente com o núcleo atômico. Nesse processo o elétron se desvia de sua trajetória inicial de 2 a $5^{\circ}$ por choque, mas sem alterar significativamente a sua velocidade e consequentemente sua energia cinética. Como resultado final desse espalhamento cerca de $30 \%$ dos elétrons incidentes emergem da superfície. Essa fração emergente é tanto maior quanto maior o peso atômico do elemento presente na superfície, como pode ser observado na Figura 3.

Uma informação bastante importante e que nem sempre é evidenciada através de imagens 


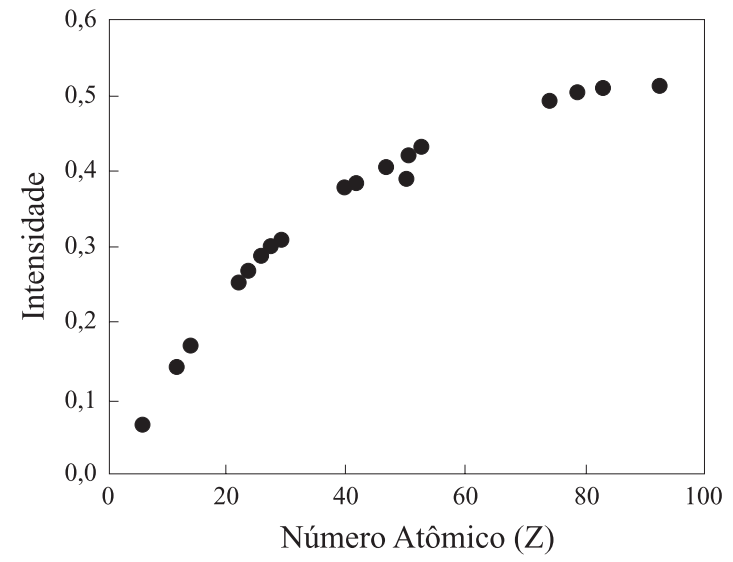

Figura 3. Intensidade de Elétrons Retro-espalhados como função do número atômico a um potencial de $20 \mathrm{kV}^{3}$

topográficas, é o posicionamento e a distribuição de elementos minerais adicionados em determinados polímeros como carga. Como a carga mineral tem um peso atômico médio superior ao da matriz polimérica, o uso do imageamento por BSE, revela pontos claros em uma matriz escura, evidenciando a presença de aglomerados com dimensões indesejadas ou distribuições heterogêneas.

\section{Análise microestrutural}

O principal objetivo da análi-

se microscópica em compostos poliméricos é a determinação da homogeneidade de dispersão de cargas na matriz e particularmente para estudo do modo ou causa da fratura. Dessa forma a MEV pode ser utilizada para investigar a zona de fratura e tentar correlacionar as feições topográficas da superfície da amostra à natureza ou causa da fratura. Imagens da superfície de fratura de um componente estrutural à base de polipropileno com carga mineral são apresentados na Figura 4 e 5. A utilização de SEI (Figura 4)

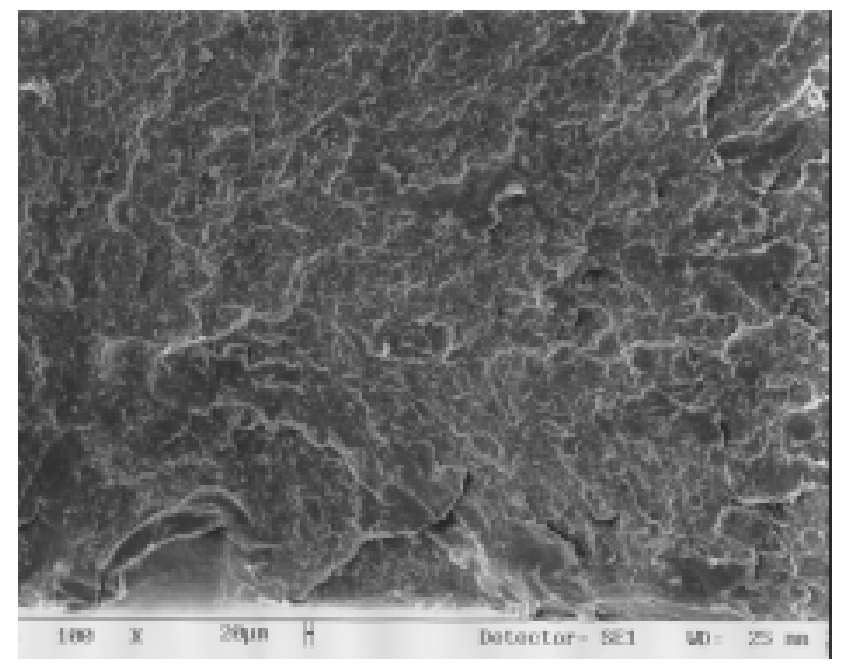

Figura 4. Utilização do detector de elétrons secundários para caracterização topográfica da superfície de fratura de um polipropileno com carga.Nota-se feições típicas de uma fratura do tipo frágil. mostra que o material sofreu fratura tipicamente frágil. Nessas condições não há contraste suficiente para se observar a dispersão de carga na matriz.

Duas situações são apresentadas para se ilustrar o uso de elétrons retroespalhados (BSE) para análise de homogeneidade de dispersão de cargas minerais em matrizes poliméricas. Na Figura 5a, imagem de BSE da superfície de fratura do componente, apresentado na Figura 4, permite a observação da dispersão das partículas de carga por contraste de número atômico uma vez que o $\mathrm{Ca}(\mathrm{Z}=21)$, principal constituinte da carga mineral, possui número atômico mais alto que o da matriz. $\mathrm{O}$ espectro de dispersão de raios-x (EDS), apresentado na Figura 6, de um aglomerado de carga da Figura 5a tem como pico mais intenso o $\mathrm{Ca}$ com alguma contaminação por Sílicio . Neste caso tanto as dimensões como a homogeneidade de dispersão da carga podem ter sido determinantes para a falha do material em serviço.

No segundo caso, Figura 5b, um filme de polipropileno reciclado a partir de garrafas de água, a imagem de BSE revela uma distribuição heterogênea de carga. O mapeamento químico (“dot mapping") desta região por dispersão de raios-x é ilustrado para o elemento Cálcio (7a), Sílicio (7b) e Magnésio (7c). Este recurso do MEV permite, ao mesmo tempo, se avaliar tanto a distribuição de carga como a sua composição química. Estas informações combinadas a imagens de SE e BSE tornam a MEV uma importante ferramenta analítica para estudo das possíveis causas de falha em compostos poliméricos. 

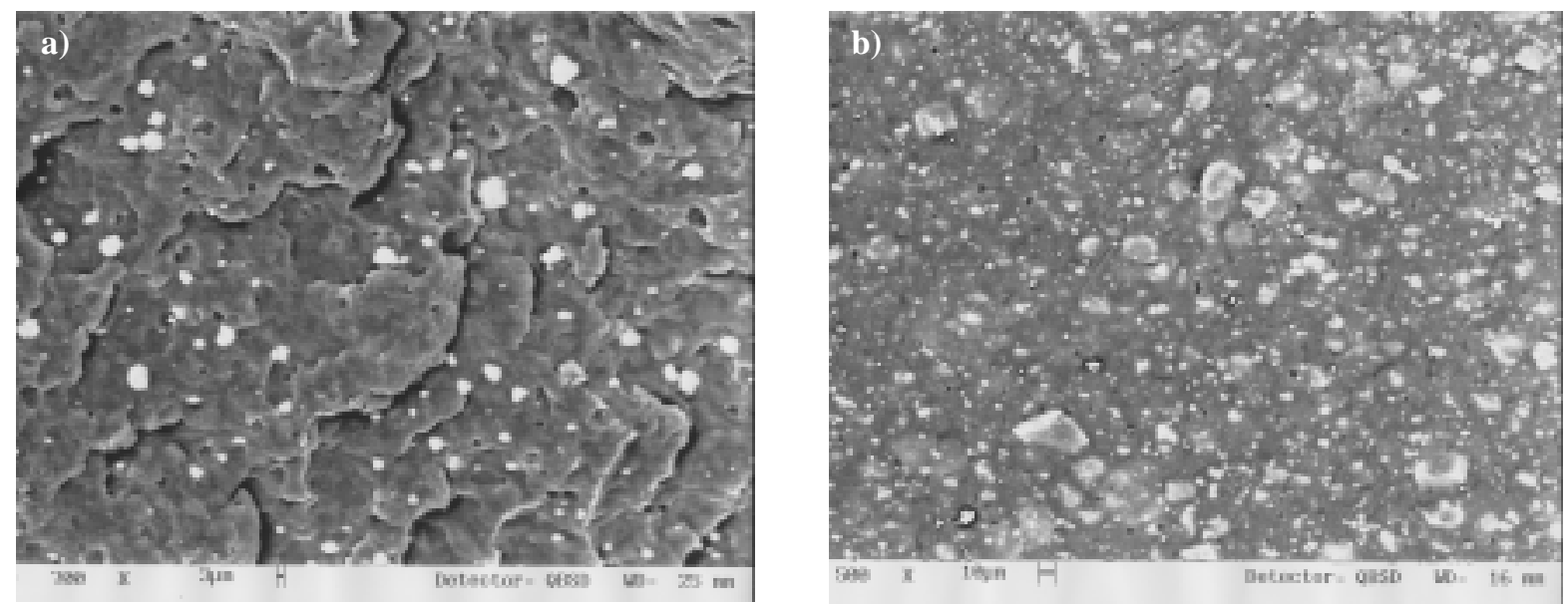

Figura 5. Imagens obtidas a partir do detector de elétrons restroespalhados mostrando adistribuição de carga mineral em superfícies de polipropileno (a) peça de engenharia apresentando aglomerados com dimensões relativamente homogêneas. (b) Filme reciclado a partir de garrafa d'água com aglomerados de dimensões heteroêneas. Essa imagem ilustra a região utilizada para os mapeamentos de raios-x apresentados na Figura 7.

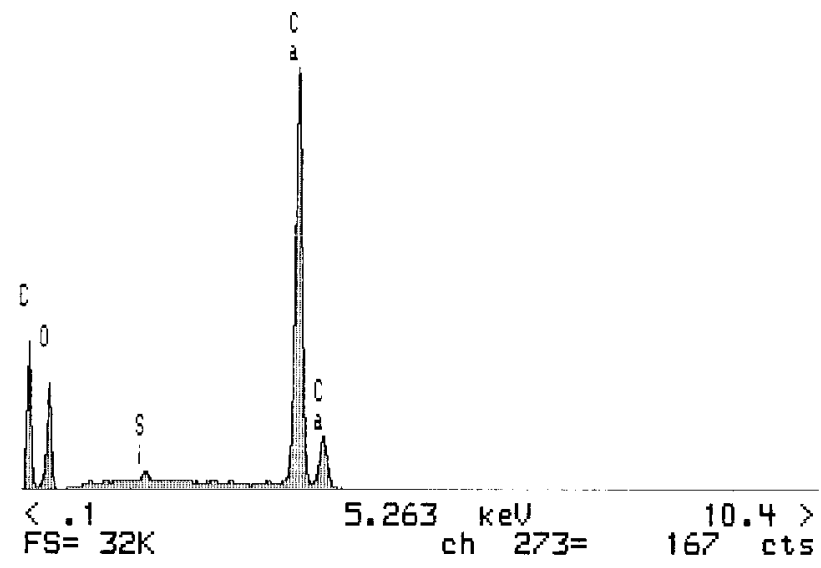

Figura 6. Espectro de dispersão deEnergia de Raios-x para uma partícula de aglomerado na superfície de fratura do polipropileno apresentado na Figura5(a). Trata-se de um carbonato de calcio com pequena contaminação por silício.
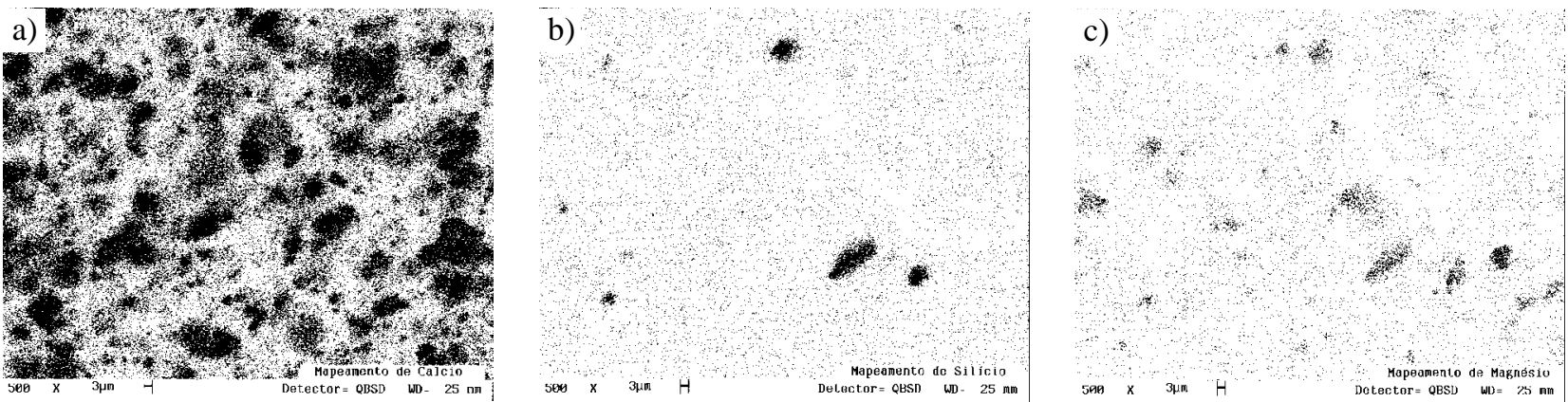

Figura 7. Mapeamentos de raios-x da superfície apresentada na Figura 5(b), mostrando em (a) o mapeamento para o elemento calcio, em (b) para o silício e em (c) para o elemento magnésio. Através desse recurso pode-se identificar quimicamente e também em termos de distribuição qualquer elemento ou contaminante presente na carga adicionada ao polímero.

\section{Referências Bibliográficas}

1. Sawyer L. C. and D. T. Grubb, Polymer Microscopy Chapman \& Hall (1987).

2. Correa, C. A. Morphological Aspects and Failure Mechanism in RubberToughened
Polystyrene. Anais da Acta Microscópica Vol 4, Supplement B, (1995).

3. Goldstein, J. I. et al. Scanning Electron Microscopy an X-Ray Microanalysis - A Textbook for Biologist, Materials Scientists and Geologists. Plenum
Press, New York (1994).

4. Williams, D.B., \& C. B. Carter, Transmission Electron Microscopy A Textbook for Materials Science. Plenum Press, New York (1996).

5.Ziebold, T.O. Anal. Chem. 39, 858 (1967).

Elaborado por Clever R. Chinaglia e Carlos A. Correa, CCDM - Centro de Caracterização e Desenvolvimento de Materiais, Universidade Federal de São Carlos, Rodovia Washington Luis, km 235, 13560-985, São Carlos, SP. 